Antony Francis Amalanesan*, Daniel Ostler, Nils Frielinghaus, Thomas Heiliger and Dirk Wilhelm

\title{
Guided capture of 3-D Ultrasound data and semiautomatic navigation using a mechatronic support arm system
}

https://doi.org/10.1515/cdbme-2020-0025

\begin{abstract}
A common treatment for malignant hepatic tumors is radiofrequency thermal ablation (RFA); however this procedure is often exhausting and error-prone, when performed only with hand held devices. In this work, we present a new concept for a computer- and mechatronicarm-assisted treatment of hepatic tumor with RFA. Challenging factors of state-of-the-art RFA procedures are tackled by utilizing a prototype, which combines state-ofthe-art ultrasound image guidance with a mechatronic support arm system. Physicians can image and examine the human abdomen using an ultrasound capturing device which is guided by the mechatronic support arm. The mechatronic arm allows to track the ultrasound transducer via joint position encoders, so that the recorded images' exact positions are acquired and the patient is automatically registered during imaging. Consequently, physicians can use the acquired ultrasound images to navigate in the imaged abdomen area. The established prototype enables preplanning and semi-automatic alignment of trajectories with sufficient accuracy. In addition, the arm system supports the usually lengthy, exhausting and error-prone holding of the needle during the ablation process. Based on the results of accuracy examination and expert assessments by surgeons, the established concept could be proven to be advantageous for the considered use case.
\end{abstract}

Keywords: holding arm system; radiofrequency thermal ablation; semiautomatic trajectory alignment; surgical assistance system; ultrasound navigation.

\section{Problem statement}

Malignant hepatic tumors and metastases are treated by resecting the cancerous part, by transplanting an entire liver, or by ablating the tumor, e.g., with radiofrequency thermal ablation (RFA). The latter method is usually performed percutaneously under ultrasound guidance and consequently minimally invasive, while the other methods are conducted laparoscopically or open-surgically. The choice of method depends on factors, like tumor size, degree of cirrhosis, tumor location, and condition of the patient, as well as on the expertise and experience of the surgeon. Especially, a percutaneous approach, as performed nowadays, requires high experience to succeed the difficult targeting in the abdomen, due to the restricted view $[2,3]$.

Percutaneous RFA procedures are preplanned, using preoperative Computed Tomography (CT) or Magnetic Resonance Imaging (MRI) images. Real-time ultrasound imaging usually guides the centric needle tip placement into the tumor. The electrodes of the RFA needle are extended to an electrode cluster, as soon as a satisfying tip position is reached. Depending on the case, the area which is covered by the electrode cluster is heated for about 8 to $25 \min [1,3]$.

Small tumors are ablated with one step, covering the entire tumor, while bigger tumors need several ablation cycles with various positionings of the needle. Follow-up examinations are usually performed with CT or MRI images [3].

Three main challenges can be seen in the state-of-theart RFA procedures: Accurate approaching of the center of the tumor is required for optimal treatment; Planning and performing of RFA on complex tumor locations arises to be difficult; Holding the needle in a fixed position during ablation is exhausting and error-prone.

\section{Approach and implementation}

The above presented challenging factors are tackled by utilizing a prototype, which combines state-of-the-art
*Corresponding author: Antony Francis Amalanesan, Research Group MITI, Klinikum rechts der Isar of Technical University of Munich, Munich, Germany; and Brainlab AG, Munich, Germany

E-mail: antony.amalanesan@tum.de

Daniel Ostler and Dirk Wilhelm, Research Group MITI, Klinikum rechts der Isar of Technical University of Munich, Munich, Germany Nils Frielinghaus and Thomas Heiliger, Brainlab AG, Munich, Germany 
ultrasound image guidance with a mechatronic support arm system (henceforth referred to as "mechatronic arm"). Physicians can examine the human abdomen using a conventional ultrasound probe which is guided by the examiner while being attached to the mechatronic arm. While capturing the images, the mechatronic arm allows to track the ultrasound transducer's position, so that the recorded images' exact spatial positions relative to the mechatronic arm are acquired. By doing this, the image set remains correlated to the patient and, consequently, the tumor, as long as the position of the mechatronic arm and the patient in relation to each other are not changed. Consequently, physicians can use these images to preplan needle trajectories and navigate in the imaged abdominal area by a semi-automatic alignment to those trajectories with sufficient accuracy, enabled by the mechatronic arm. In addition, the mechatronic arm supports the holding of the needle during the ablation process.

\section{Hardware setup}

The hardware setup for the solution is composed of (1.) a mechatronic arm with a mounting part for defined attaching of an ultrasound transducer, (2.) an ultrasound unit, (3.) a frame-grabber and (4.) a computer (Figure 1).

The mechatronic arm manufactured by Medineering $\mathrm{GmbH}$ (Munich, Germany) and marketed by Brainlab AG (Munich, Germany) as Brainlab Cirq ${ }^{\circledR}$ for cranial and spinal interventions is used in this project for guiding and navigation purposes. The arm has seven rotational joints, which can individually be fixed into a specific position by controlling the brakes installed in each of the joints. Consequently, the arm can be moved manually while the brakes are released and fixed in a specific position by activating the brakes. In addition, the brakes can be controlled individually through HTTP-requests via an Ethernet interface; This feature is applied for the semiautomatic trajectory alignment within the implementation. Furthermore, the mechatronic arm features resolvers, which are installed in the joints and return the current joint position, and buttons, which are by default used to control the brakes, but also return specific values. The arm is securely mounted to the rail of an operation table using the mounting mechanism. A hand part manufactured by Brainlab $A G$, which allows to attach any kind of mounting parts with the specific interface can be docked on the front interface of the arm.

The mounting parts for defined attaching of the ultrasound transducers and guiding the ablation needle are manufactured additively using the FDM-printer Stratasys

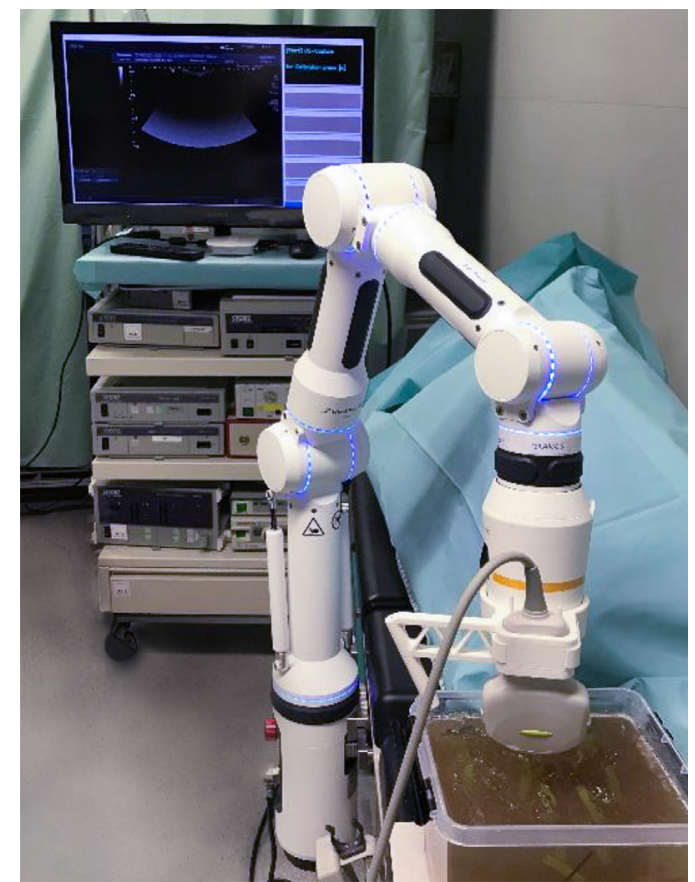

Figure 1: Setting for validation experiments; mechatronic arm with attached transducer in the foreground, monitor displaying the user interface in the background.

Fortus 250mc produced by Stratasys (USA) and ABS filaments (ABS-P430 for model, ABS-SR30 for Support) with a slice height of $0.1778 \mathrm{~mm}$. For designing purposes, the software PTC Creo Parametric 2.0 developed by PTC (USA) is used.

The geometry of the transducer was considered for the design, so that an exactly-fitting clamping mechanism was designed and prototyped to match the transducer geometry. Consequently, the position and tilt of the transducer tip were given by geometry. The needle guide enables directing the needle in the image plane in a specific tilt towards the middle of the image.

For ultrasound imaging purposes during the final accuracy tests, the ultrasound unit Xario ${ }^{T M} 2000$ and the convex transducer for abdominal, fetal and pediatric imaging PVU-375BT, both marketed by Canon Medical (Japan, formerly known as Toshiba Medical), are used.

To capture and convert the ultrasound images, the frame-grabber XI1OOXUSB-PRO by Magewell (China) is used. Provided with ultrasound images via DVI-input, it forwards the captured images to the computer via USB.

The application is executed on a laptop with an installation of Python 3.6 and required libraries. Furthermore, an external monitor is connected to display the user interface. 


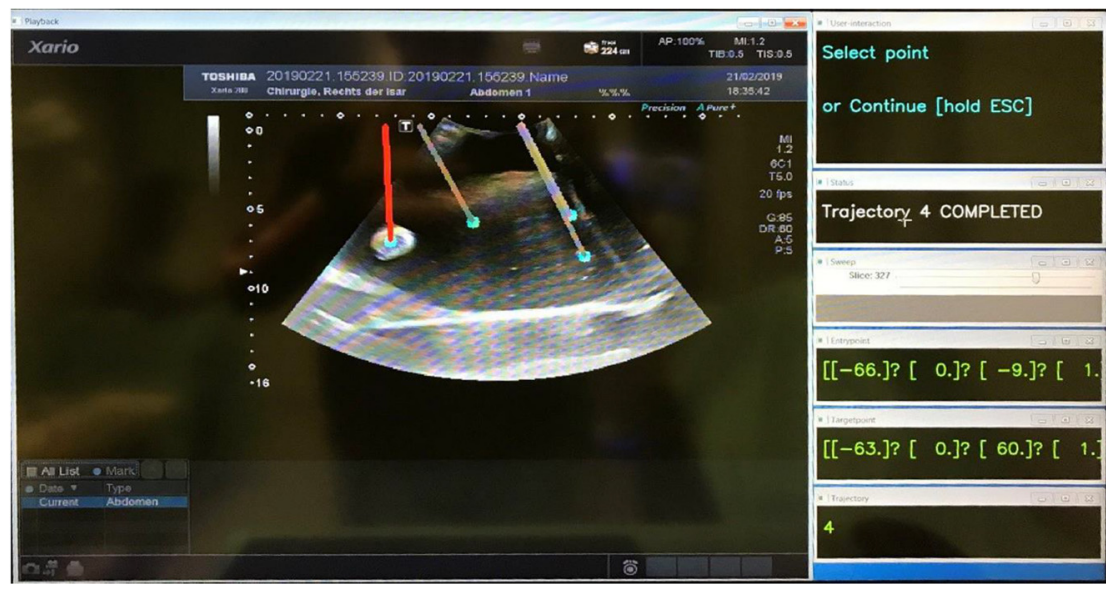

Figure 2: Trajectory planning on the user interface: In the main window, the currently selected image slice and planned trajectories are visible. The trajectory laying in the current image's plane is highlighted in red. The windows on the left side display user interaction requests, status messages and coordinate information of currently planning trajectory. The slide bar enables switching between image slices.

\section{Software implementation}

The entire workflow is implemented in a modular Python 3.6 architecture. The GUI (Figure 2) is established using the Python library OpenCV 3.4.3. It guides the user through the workflow and enables appropriate interactions.

\section{Guided ultrasound capture}

The mechatronic arm guidance enables a mechanical tracking of the ultrasound transducer, which is firmly mounted to the front interface of the arm. A software module is implemented to capture the ultrasound images and concurrently track the location and orientation of the transducer tip, using the acquired information from the resolvers of all arm joints and the kinematics of the arm. In this way, continuously captured ultrasound images are stored together with the corresponding positions in the spatial environment and made available for the upcoming steps.

\section{Semi-automatic navigation}

The semi-automatic navigation in the acquired images includes a preplanning step and a trajectory approaching step:

For the preplanning step, the implementation allows physicians to play back and observe the acquired images and to plan several needle trajectories by defining appropriate entry- and target points. The pathway of the trajectory can be assessed in the entire set of the images by scrolling through the image set, to check and adjust the trajectory, e.g., to preempt possible injuries of risky structures.

In the positioning and approaching step, the implementation allows physicians to switch between and inspect the preplanned trajectories, and select the desired one. Subsequently, the program determines the joint angles required to approach the selected trajectory, using inverse kinematics calculations. Afterward, the joints of the mechatronic arm are automatically unlocked one after one, and the physician is asked to set the required joint angle by manually moving the arm. As soon as the required joint position is reached, the currently open joint is automatically locked down and the next joint is unlocked to be set. By setting all joints of the arm to the required joint angles, the selected trajectory is approached. The software determines the actually approached position using the kinematics and the resolver data, and calculates and presents the deviation from the desired trajectory. Based on the sufficiency of the acquired precision, the physician can confirm appropriate positioning and continue to the next step, or deny the sufficiency and request positioning again.

\section{Holding task}

If the trajectory is confirmed, the software switches to the holding program, where all brakes of the arm are blocked and the GUI is showing the real-time ultrasound image on screen. From now on, the usual RFA procedure is followed, simply supported by the mechatronic arm. The physician can penetrate the ablation needle in the direction of the trajectory by using the available needle guide, while monitoring the incision and the ablation process.

\section{Accuracy assessment}

\section{Material and methods}

Final validation experiments and demonstrations are performed on ultrasound phantoms made of gelatin. Olives, which are placed inside the phantom, represent liver tumors. 

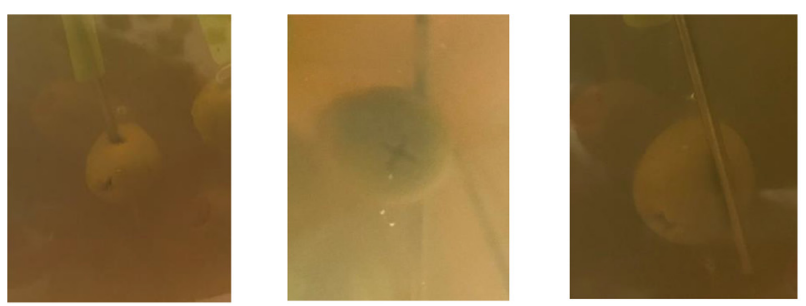

In total, 51 punctures were performed by various test persons (medical and non-medical background). The test persons were introduced to the system through a demonstration of the complete workflow. After a trial ultrasound scan on the phantom, to get familiar with the system, the test person is asked to run through the entire workflow. This includes scanning the phantom and locating the olives, capturing appropriate sweeps, planning trajectories targeting the center of the olives, approaching the trajectories, and puncturing the olives through the guide. Puncture needles having a round cross-sectional area with a diameter of $2.4 \mathrm{~mm}$, peaked ends and a length of about $30 \mathrm{~cm}$ are used for the examinations. The whole workflow is validated at once, thus different parts are not validated individually.

The test persons are asked to try out two variations of capturing: Static capturing, where the images are recorded for one olive, while the mechatronic arm is fixed in a position; and dynamic capturing, where several olives are scanned in a dynamic way. These variations are later compared considering the puncture accuracy.

After the puncture, photos of the results, where the position of the needle in relation to the olive can be assessed visually (Figure 3) are taken and measurements on the ultrasound unit are performed, to evaluate the centric approach of the olives. Hereby, the olive was marked with four points on the edge and the lengthwise and crosswise thickness of the visible cross section was gauged. The needle pathway was marked with further two points.

The image manipulation software Inkscape was applied for evaluation purposes. An ellipse was defined on the olive cross section, using the four defined points, and a line was defined on the needle, using the two defined points on the visible pathway. The centricity of the needle was assessed by measuring the distance of the line from the center of the olive, i.e., the ellipse. Therefore, a line perpendicular to the needle pathway was defined and the distance to the intersection points with the ellipse edge was measured.

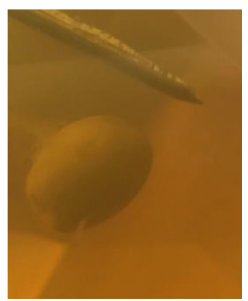

Figure 3: Photographs of olive punctured centrically, eccentrically (penetrating flesh or penetrating shell), and olive missed.
In cases, the test person was a surgeon, they were asked to commend on the usability of the guided imaging and user interface, as well as potential advantages of the present concept for RFA interventions.

\section{Results}

In 50 cases of the 51 punctures, the olive could be relocated and punctured through the needle guide. In 25 cases among them, the olive was punctured centrically, while in the remaining cases the olive was punctured visibly eccentrically. The average deviation of the punctures from the center, determined from the measurements on the ultrasound unit, amounted to $2.87 \mathrm{~mm}$, whereby dynamically performed sweeps show a higher eccentricity with an average of $3.87 \mathrm{~mm}$. A potential reason for this is probably the latency between image capturing and corresponding location data processing.

Surgeons confirmed the advantages of the holding-, capturing- and navigation features for the present use case. Needs of improvement have been identified in the user friendliness of the GUI and the handling of the mechatronic-arm-guided ultrasound probe.

\section{Conclusion}

The results for approaching the preplanned trajectories show high reliability and sufficient accuracy for the considered use case. Allowing real-time tracking of the puncture, small inaccuracies can be identified and adjusted manually.

Overall, we were able to successfully introduce a new concept for a computer- and mechatronic-arm-guided RFA, exhibiting adequate accuracy results and satisfying endorsement of the advantages by experts.

Research funding: The author state no funding involved. Author contributions: All authors have accepted responsibility for the entire content of this manuscript and approved its submission. 
Competing interest: Corresponding author and two coauthors are affiliated with Brainlab AG, the company marketing the mechatronic arm system used for this study.

Informed consent: $\mathrm{n} / \mathrm{a}$.

Ethical approval: The conducted research is not related to either human or animals use.

\section{References}

1. Kuvshinoff BW, Ota DM. Radiofrequency ablation of liver tumors: influence of technique and tumor size. Surgery 2002;132;S605-12.

2. Manns MP, Schneidewind S. Praxis der Hepatologie. Berlin, Heidelberg: Springer; 2016.

3. McGahan JP, Dodd III GD. Radiofrequency ablation of the liver: current status. Am J Roentgenol 2001; 176: S3-16. 